\title{
Assessing the building envelope performance during occupancy
}

\author{
Jade Deltour ${ }^{1}$, Nicolas Heijmans ${ }^{1}$, and Karel De Sloover ${ }^{1}$ \\ ${ }^{1}$ BBRI, Energy Aspects of Buildings Laboratory, 1342 Limelette, Belgium
}

\begin{abstract}
In order to achieve carbon neutrality in the building field as expected by the Energy Performance of Buildings Directive, it is important to not only be able to calculate energy performance during design but also to be able to measure the actual energy performance of buildings during occupancy. However, there is currently no approved methodology for assessing the energy performance of the building envelope of an occupied building independently of its occupants, its systems and the climate. We applied a mathematical method to determine the heat loss coefficient (HLC) of the building envelope, from data collected in occupied buildings. The paper describes the in-situ measurement protocol and the mathematical models that contributes to address this challenge. Our methodology is demonstrated on a new semi-detached house, more insulated than the regulatory level, located in Brussels from a full year of monitoring data. For this case study, the results are promising. Indeed, some mathematical models show results of the same range value for the occupied insitu HLC and the reference HLC, obtained from in-situ measurements but without occupancy. However, more case studies should be evaluated, in order to validate the methodology.
\end{abstract}

\section{Introduction}

\subsection{Context}

In order to achieve carbon neutrality in the building field as expected by the Energy Performance of Buildings Directive, it is important not only to calculate energy performance during design but also to measure the actual energy performance after completion.

In most cases, a building's energy performance is assessed by calculation, this theoretical consumption is often compared to the measured consumption. However, various factors can distort this comparison, namely:

- the actual use of the building

- the climate

- the quality of the building's execution

- the setting and the maintenance of the HVAC systems

- the initial rate of relative humidity in the constructed building.

As the intended goal is to reduce the actual energy consumption rather than the theoretical consumption, it may prove useful to measure the intrinsic performance of the building (the building envelope, with or without the HVAC systems) regardless of the occupancy of the building and the climate. This measurement offers numerous benefits. For instance, it can:

- help the building professionals to better assess the impact of certain choices (related to design and execution)
- provide an indication of the quality of the work carried out, which could increase the trust between clients and construction companies

- in the long term, guarantee performances, regardless of the behavior of users

\section{$1.2 \quad$ Link with building physics}

The physical approach used to determine the thermal performance of the building envelope based on in-situ measured data is described below.

The heat loss coefficient (HLC) is the indicator of the thermal performance of the building envelope. This coefficient takes into account the heat losses due to both transmission (via the walls) and infiltration (via air leaks).

$$
\mathrm{HLC}=H_{t r}+H_{\text {inf }}
$$

where:

$\mathrm{H}_{\text {tr }}$ is the heat loss by transmission $[\mathrm{W} / \mathrm{K}]$

$\mathrm{H}_{\text {inf }}$ is the heat loss by infiltration [W/K].

On the other hand, a simplified building's heat balance equation can be written as follows if the latent heat effects are neglected [1]:

$$
C_{i} \frac{\partial \mathrm{T}_{\mathrm{i}}}{\partial \mathrm{t}}=\Phi_{\mathrm{Ph}}+\Phi_{\mathrm{int}}+\Phi_{\mathrm{sol}}+\underbrace{\Phi_{\mathrm{tr}}+\Phi_{\mathrm{inf}}}_{\alpha H L C}+\Phi_{\mathrm{vent}}
$$

where:

- $\mathrm{C}_{\mathrm{i}}$ is the effective averaged heat capacity of the dwelling

\footnotetext{
* Corresponding author: jade.deltour@bbri.be
} 
- $\mathrm{T}_{\mathrm{i}}$ is the average indoor temperature of the dwelling

- $\Phi$ is the the heat flux from:

- Ph: heating system - int: internal gains

- sol: solar gains _ - tr: transmission

- inf: infiltration - vent: ventilation system

Some of these heat flux can be described by the following physical equations:

$$
\begin{gathered}
\Phi_{\text {sol }}=g A \cdot \mathrm{I}_{\text {sol }} \\
\Phi_{\text {tr }}=H_{t r} \cdot\left(\mathrm{T}_{\mathrm{e}}-\mathrm{T}_{\mathrm{i}}\right) \\
\Phi_{\text {inf }}=H_{\text {inf }} \cdot\left(\mathrm{T}_{\mathrm{e}}-\mathrm{T}_{\mathrm{i}}\right) \\
\Phi_{\text {vent }}=\dot{m} \cdot c_{\mathrm{a}} \cdot\left(\mathrm{T}_{\text {vent }}-\mathrm{T}_{\mathrm{i}}\right)
\end{gathered}
$$

where:

- $\mathrm{gA}$ is the solar heat gain factor

- $\mathrm{I}_{\text {sol }}$ is the solar radiation

- $\mathrm{T}_{\mathrm{e}}$ is the average outdoor temperature

- $\mathrm{T}_{\mathrm{i}}$ is the average indoor temperature

- $\mathrm{T}_{\text {vent }}$ is the pulsed air temperature

- $\dot{\mathrm{m}}$ is the total supply flow rate

- $\mathrm{c}_{\mathrm{a}}$ is the air heat capacity

By combining equations (1), (4) and (5), we have:

$$
\Phi_{\text {tr }}+\Phi_{\text {inf }}=H L C .\left(\mathrm{T}_{\mathrm{e}}-\mathrm{T}_{\mathrm{i}}\right)
$$

\section{Methodology}

Currently, the heat loss coefficient HLC can be obtained by applying different measurement protocols. In this paper, the following 3 protocols are described:

- the (regular) co-heating test

- an adapted co-heating test

- an on-board monitoring

For each protocol, the methodology is the same and organized in 2 steps:

1. Data collection

2. Data analysis

Results obtained from these protocols can be compared with a theoretical HLC as explained in the last paragraph of this section.

\section{1 (Regular) co-heating test}

This test is quoted as "regular" as this is the most common co-heating test currently performed and as is currently being standardized [2].

\subsubsection{Data Collection}

During the regular co-heating test, the indoor temperature of the building is maintained at approximately $25^{\circ} \mathrm{C}$ with the help of a dedicated electrical heating system. The test must be carried out in an empty building over a fifteen-day period, during the heating season, i.e. when there are low levels of solar radiation.

The following measurements are recommended:

- Heating power in each heated room - Ph (W)

- Internal temperature and relative humidity in each room $-\mathrm{T}_{\mathrm{i}}\left({ }^{\circ} \mathrm{C}\right)$ and $\mathrm{RH}(\%)$

- Electric consumption of the circulation fans (kWh)

- External temperature $-\mathrm{T}_{\mathrm{e}}\left({ }^{\circ} \mathrm{C}\right)$

- Solar radiation $-\mathrm{I}_{\mathrm{sol}}\left(\mathrm{W} / \mathrm{m}^{2}\right)$

- Wind speed $(\mathrm{m} / \mathrm{s})$

Relative humidity is only necessary when building is not sufficiently dried-out prior to the commencement of the test. And wind speed is, e.g., necessary to separate heat loss due to the air infiltration from heat loss due to transmission. Moreover, it is recommended to meet the following requirements [3] during the test in order to guarantee a reliable and reproducible result:

- Building functionally must be completed and suitable for occupancy

- A fan pressurization test must be completed before or after the test

- All intended ventilation openings must be closed or sealed

- Internal lights and appliances must be switched off

- Measurements must be made in each habitable room

- Homogeneous internal temperature: $\pm 1 \mathrm{C}^{\circ}$ of the mean internal set point temperature

- Internal temperature set between 20 and $25^{\circ} \mathrm{C}$ and must not exceed $30^{\circ} \mathrm{C}$ (due to solar radiation)

- Daily mean of $\left(\mathrm{T}_{\mathrm{e}}-\mathrm{T}_{\mathrm{i}}\right)$ must be at least $10 \mathrm{~K}$

- Logging intervals: $\min 1 \mathrm{~min}-\max 10 \mathrm{~min}$

- $\quad \ldots$

\subsubsection{Data analysis}

The average method is detailed in the ISO 9869-1 standard. This method was initially developed to be applied on walls, not on buildings.

By generalizing this method to buildings, it assumes that the HLC can be obtained by dividing the mean of the heat power measurements by the mean temperature difference. If the index $\mathrm{j}$ indicates measurements, then an estimate of the HLC is obtained by

$$
H L C=\frac{\sum_{j=1}^{n} P_{h, j}}{\sum_{j=1}^{n}\left(T_{i, j}-T_{e, j}\right)}
$$

Indeed, this method neglects the dynamic behavior, and the heat balance equation (2) becomes:

$$
0=\Phi_{\mathrm{Ph}}+\Phi_{\mathrm{int}}+\Phi_{\mathrm{sol}}+\Phi_{\mathrm{tr}}+\Phi_{\mathrm{inf}}+\Phi_{\mathrm{vent}}
$$

Considering that $\Phi_{\text {int }}$ and $\Phi_{\text {vent }}$ are both equal to zero due to data collection conditions and that $\Phi_{\text {sol }}$ can be assumed to be negligible during the heating season (low solar radiations), the heat balance can be reduced to:

$$
0=\Phi_{\mathrm{Ph}}+\Phi_{\mathrm{tr}}+\Phi_{\mathrm{inf}}
$$


And combining with (7),

$$
\Phi_{\mathrm{Ph}}=H L C \cdot\left(\mathrm{T}_{\mathrm{i}}-\mathrm{T}_{\mathrm{e}}\right)
$$

An initial study [4] has shown that the degree of uncertainty concerning the result is approximately $8 \%$ if the entire protocol is followed.

Various options for improvement are currently being investigated in order to increase the scope of application of this measurement method as much as possible:

1. reduction of the test duration (adapted co-heating)[5]

2. performing measurements even with occupancy [6]

3. use of the heating system of the building itself (integrated co-heating) [7]

4. use of connected and wireless measuring devices

5. performing measurements regardless of the season [8] The first 2 improvements are described in the following protocols.

\subsection{Adapted co-heating test}

\subsubsection{Data Collection}

During that test, instead of setting the indoor temperature at $25{ }^{\circ} \mathrm{C}$, the heating system is subjected to on/off cycles, lasting 4 to 8 days (Fig. 1).

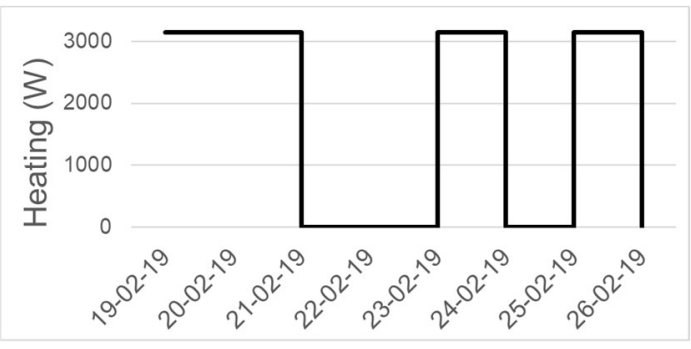

Fig. 1. Heating signal

The parameters to be measured are the same as for the regular co-heating test: $\mathrm{Ph}(\mathrm{W}), \mathrm{T}_{\mathrm{i}}\left({ }^{\circ} \mathrm{C}\right), \mathrm{RH}(\%)$, circulation fans $(\mathrm{kWh}), \mathrm{T}_{\mathrm{e}}\left({ }^{\circ} \mathrm{C}\right), \mathrm{I}_{\mathrm{sol}}\left(\mathrm{W} / \mathrm{m}^{2}\right)$ and wind speed $(\mathrm{m} / \mathrm{s})$.

All the recommendations explained for the regular test remain applicable. However, due to the on/off cycles of the heating, it is harder to maintain a homogeneous internal temperature across all habitable rooms.

\subsubsection{Data analysis}

Due to dynamic signal controlling the heating system, assumptions made for the average method cannot be applied with the adapted co-heating test protocol. Consequently, a more advanced data analysis method is used, based on a so-called RC model.

In building physics, RC models link the thermal variables of the building by a network composed of resistances and capacitances (e.g. multi-zone model). This implies that, usually, the parameters have a direct physical interpretation. This allows the use of prior physical knowledge of the building to be incorporated into the model [1], [6].

For our purpose, very simple RC models are used (e.g. Fig. 2). Depending on the building and the climatic conditions encountered, different RC models should be developed.

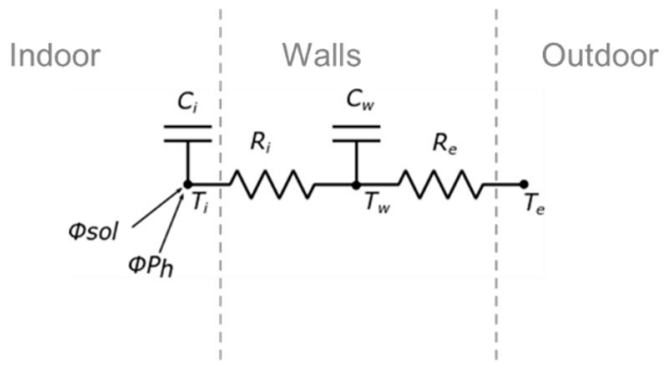

Fig. 2. RC model example for adapted co-heating

In the model depicted in Fig. $2, \mathrm{R}_{\mathrm{i}}$ and $\mathrm{R}_{\mathrm{e}}$ represent, the thermal resistances of the walls and the losses through infiltration. $\mathrm{C}_{\mathrm{i}}$ and $\mathrm{C}_{\mathrm{w}}$ are, respectively, the heat capacity inside the building and of the external walls. $T_{i}, T_{w}$ and $T_{e}$ are, respectively, the indoor temperature, the temperature of the wall and the outdoor temperature.

Thus, the HLC is the inverse of the sum of both resistances.

$$
\mathrm{HLC}=\frac{1}{\left(\mathrm{R}_{\mathrm{i}}+\mathrm{R}_{\mathrm{e}}\right)}
$$

The following equations are the mathematical expressions of the RC model (Fig. 2.)

$$
\begin{gathered}
\frac{\mathrm{dT}_{\mathrm{i}}}{\mathrm{dt}}=\frac{\left(\mathbf{T}_{\mathbf{w}}-\mathbf{T}_{\mathbf{i}}\right)}{R_{i} \cdot C_{i}}+\frac{\Phi_{P h}}{C_{i}}+\frac{\Phi_{\text {sol }}}{C_{i}} \\
\frac{\mathrm{dT}_{\mathrm{w}}}{\mathrm{dt}}=\frac{\left(\mathbf{T}_{\mathbf{i}}-\mathbf{T}_{\mathbf{w}}\right)}{R_{i} \cdot C_{w}}+\frac{\left(\mathbf{T}_{\mathbf{e}}-\mathbf{T}_{\mathbf{w}}\right)}{R_{e} \cdot C_{e}}
\end{gathered}
$$

Equations 13 and 14 should be solved using an optimization tool in order to identify the parameters $\mathrm{R}_{\mathrm{i}}$, $R_{e}, C_{i}$ and $C_{w}$, based upon the measurements of $T_{i}, T_{e}, I_{s o l}$ and $\Phi_{\mathrm{Ph}}$.

\subsection{On-board monitoring}

\subsubsection{Data Collection}

For this protocol, measurements are performed regardless of occupancy. This means that the duration of the test is no longer a limitation, provided the sensors are discreet enough.

Most of the parameters to be measured are the same as for the regular co-heating test: $\mathrm{Ph}(\mathrm{W}), \mathrm{T}_{\mathrm{i}}\left({ }^{\circ} \mathrm{C}\right), \mathrm{RH}(\%)$, $\mathrm{T}_{\mathrm{e}}\left({ }^{\circ} \mathrm{C}\right), \mathrm{I}_{\mathrm{sol}}\left(\mathrm{W} / \mathrm{m}^{2}\right)$ and wind speed $(\mathrm{m} / \mathrm{s})$.

However, the internal gains and the heat loss due to ventilation can no longer be neglected. Thus, the following additional parameters should be, ideally, measured:

- Total electricity consumption and, if possible, electricity consumption for lighting

- Consumption of cold and hot water 
- Presence indicator $\left(\mathrm{CO}_{2}\right.$, infrared detector, $\left.\mathrm{RH}, \ldots\right)$

- Flow rate of the ventilation system

- Pulsed air temperature and, if possible, extracted air temperature.

Heat gains from occupants can be estimated by different approaches. E.g., an infrared detector can give an estimation of the number of occupants in a room. This number can then be converted in $\mathrm{W}$ according to the ISO 8996 [9] standard.

\subsubsection{Data analysis}

Similarly to the adapted co-heating test, the dynamic nature of the data can only be analyzed by advanced data analysis methods. Again, RC models are used. However, additional heat fluxes from internal gains and ventilation must be included in the model (Fig. 3).

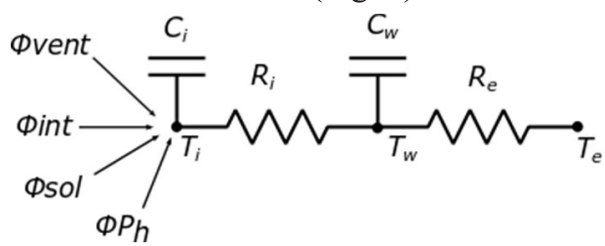

Fig. 3. RC model example for on board monitoring

Thus, the mathematical expressions of the RC model become:

$$
\begin{gathered}
\frac{\mathrm{dT} \mathrm{T}_{\mathrm{i}}}{\mathrm{dt}}=\frac{\left(\mathbf{T}_{\mathbf{w}}-\mathbf{T}_{\mathbf{i}}\right)}{R_{i} \cdot C_{i}}+\frac{\Phi \mathrm{Ph}}{C_{i}}+\frac{\Phi \text { sol }}{C_{i}}+\frac{\Phi \text { int }}{C_{i}}+\frac{\text { Фven }}{C_{i}} \\
\frac{\mathrm{dT}_{\mathrm{w}}}{\mathrm{dt}}=\frac{\left(\mathbf{T}_{\mathbf{i}}-\mathbf{T}_{\mathbf{w}}\right)}{R_{i} \cdot C_{w}}+\frac{\left(\mathbf{T}_{\mathbf{e}}-\mathbf{T}_{\mathbf{w}}\right)}{R_{e} \cdot C_{e}}
\end{gathered}
$$

As explained in 2.3.1, the heat gains from occupants can be estimated by different approaches. In our research, we decided to establish an occupancy profile based on $\mathrm{CO}_{2}$ measurements. Other studies [10] have shown the evolution of $\mathrm{CO}_{2}$ to be a reliable indicator of the presence of occupants.

The conversion from the occupancy profile to the heat gain from the occupants is determined directly by the RC model. Indeed, $\Phi_{\text {int }}$ can be expressed as follow:

$$
\Phi_{\text {int }}=k \text {. Users }
$$

Where $k$ is the heat released by the occupants (in W) and Users is an occupancy profile. $k$ is thus another parameter to be identified by the RC model, based upon the measured data including Users.

The Users occupancy profile is established through a detection algorithm based on $\mathrm{CO}_{2}$ concentration and its change over time. The algorithm takes place in two steps, and identifies 4 states: $0,0.25,0.75$ and 1 .

During the first step, a binary on/off profile $(0 / 1)$ is established based solely on the evolution of the $\mathrm{CO}_{2}$ concentration. The goal of the second step is to mitigate those moments where a change of occupancy is detected for an extremely small span of time, using 2 intermediate states:

- 0.25: intermediate state where 1 was established in step 1, but adjacent timesteps are 0 (before and after)
- 0.75: intermediate state where 0 was established in step 1, but adjacent timesteps are 1 (before and after)

The full algorithm is:

1. First step: on/off profile is established for each timestep $\mathrm{t}$ :

- IF $\mathrm{CO}_{2(\mathrm{t}-1)}<\mathrm{CO}_{2(\mathrm{t})}<\mathrm{CO}_{2(\mathrm{t}+1)}$, THEN Users $(\mathrm{t})=1$

- ELSE, IF $\mathrm{CO}_{2(\mathrm{t}-1)}<\mathrm{CO}_{2(\mathrm{t})}$ and $\mathrm{CO}_{2(\mathrm{t}+1)}<\mathrm{CO}_{2(\mathrm{t}+2)}$ and $\frac{\mathrm{CO}_{2(t+1)-} \mathrm{CO}_{2(t)}}{\mathrm{CO}_{2(t)}}<0.5 \%$, THEN Users $(\mathrm{t})=1$

- ELSE, IF $\mathrm{CO}_{2(\mathrm{t}-1)}>\mathrm{CO}_{2(\mathrm{t})}$ and $\mathrm{CO}_{2(\mathrm{t})}<\mathrm{CO}_{2(\mathrm{t}+1)}$, $\operatorname{THEN~Users}_{(\mathrm{t})}=1$

- ELSE, Users $_{(\mathrm{t})}=0$

2. Second step: detection of intermediate states

- IF Users $_{(\mathrm{t})}=1$ AND Users $(\mathrm{t}-1)=\operatorname{Users}_{(\mathrm{t}+1)}=0$, THEN Users $_{(\mathrm{t})}=0.25$

- $\quad$ IF Users $_{(\mathrm{t})}=0$ AND Users $\mathrm{At}_{(\mathrm{t}-1)}=\operatorname{Users}_{(\mathrm{t}+1)}=1$, THEN Users $_{(\mathrm{t})}=0.75$

\subsection{Theoretical HLC.}

It is relevant to compare the measured value with a reference value. Therefore, a theoretical calculation to determine the reference value is used.

The HLC is decomposed in 2 elements.

$$
\mathrm{HLC}=H_{\text {inf }}+H_{t r}
$$

The infiltration losses are determined by:

$$
H_{\text {inf }}=0.34 *\left(\frac{\mathrm{n}_{50} * \text { vol.int. }}{25}\right)
$$

Where $n_{50}$ is the air leakage (ach at $50 \mathrm{~Pa}$ ) and vol. int is the interior volume.

The transmission's losses are determined by:

$$
H_{T R}=\left(\sum U_{i} \cdot S_{i} \cdot b_{i}\right) \cdot(1+f)
$$

Where $U$ is the thermal transmittance of the exterior walls, $\mathrm{S}$ the wall surface, $\mathrm{b}$ a weighting factor for walls in contact with unheated spaces. Ideally, the impact of thermal bridges should be evaluated on basis of the plans, but this is not always possible (no plan available, lack of time). And $\mathrm{f}$ is a default factor (according to building insulation and compacity) to include the transmission losses due to thermal bridges.

\section{Case study}

\subsection{House description}

\subsubsection{General information}

The case is a high insulated semi-detached house located in Brussels, distributed by an open staircase over 4 floors including 1 semi-buried floor (Fig. 4). 


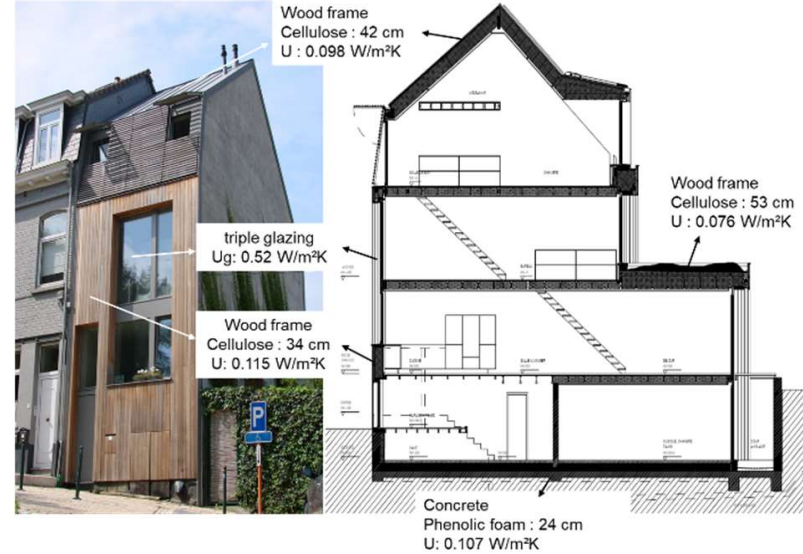

Fig. 4. House picture and section

The following characteristics give some indicative information:

- Construction year: 2010

- Occupants: couple with a regular guest

- Heated floor area: $153.7 \mathrm{~m}^{2}$

- Loss surfaces (external dimension): $521.9 \mathrm{~m}^{2}$

- All thermal bridges were optimized and calculated according to the Passive House certification

- Interior volume: $400.4 \mathrm{~m}^{3}$

- Airtightness: $0.52 \mathrm{~h}^{-1}$ measured at $50 \mathrm{~Pa}\left(\mathrm{n}_{50}\right)$

- Averaged U value (including external walls and windows): $0.23 \mathrm{~W} /\left(\mathrm{m}^{2} . \mathrm{K}\right)$

- Theoretical reference value of HLC: $94.2 \mathrm{~W} / \mathrm{K}$

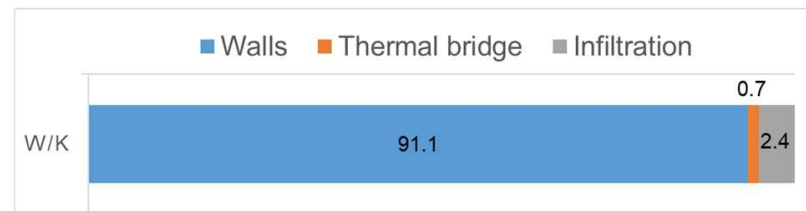

Fig. 5. Theoretical distribution of the losses included in the reference value of HLC

\subsubsection{Systems}

Heating is provided by a single heating system, a gas insert, located in the living room, with maximum power of $9 \mathrm{~kW}$ and theoretical efficiency of $85 \%$.

Domestic hot water (DHW) is provided by:

- an electric storage water heater of 10 liters located in the garage, used for the kitchen and the wash basin in the WC at first floor,

- an instant gas water heater combined with a thermal solar panel $\left(2 \mathrm{~m}^{2}\right)$ for the bathroom.

Ventilation is provided by a balanced mechanical system with heat recovery with a theoretical efficiency of $84 \%$.

\subsection{Applied protocols}

In February 2019, an "adapted" co-heating was carried out during the occupants' holidays. This test should result in a reference HLC, measured without the noise caused by occupancy.

Moreover, a full monitoring was carried out as part of the Exemplary Building certification (BATEX [11]), between October 2015 and October 2016. The resulting data was made available by the certification body to support our research. This allows us to assess the possibility of using on-board data to establish the HLC.

\subsubsection{Adapted co-heating}

Fig. 1 shows the heating signal used in the house during the adapted co-heating test.

The following parameters were monitored, each 5 min, over a period of 7 days with the equipment shown in Fig. 6:

- Heating power $\mathrm{Ph}(\mathrm{W})$

- Indoor temperatures in each habitable room $\mathrm{T}_{\mathrm{i}}\left({ }^{\circ} \mathrm{C}\right)$

- Exterior temperature $\mathrm{T}_{\mathrm{e}}\left({ }^{\circ} \mathrm{C}\right)$

- Global horizontal solar irradiation $\mathrm{I}_{\mathrm{sol}}\left(\mathrm{W} / \mathrm{m}^{2}\right)$

Note: some of the recommended parameters were not recorded because the limitations of the measuring kit.

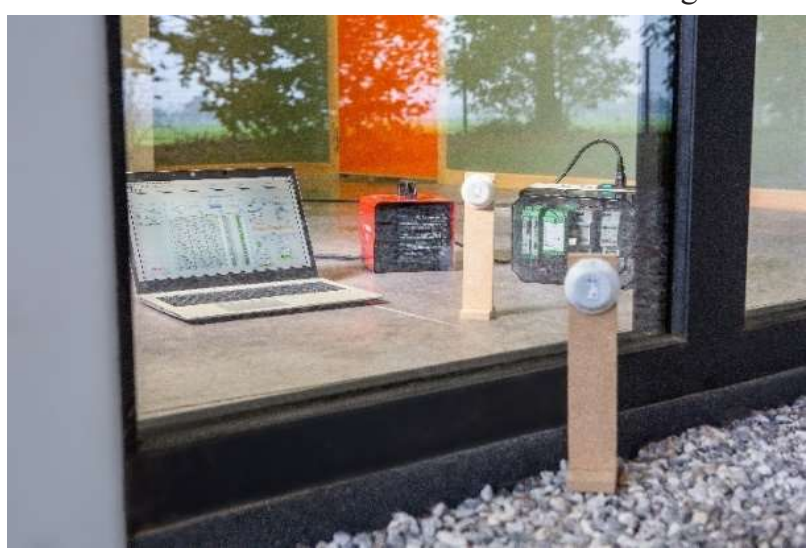

Fig. 6. BBRI electric heating kit

Before the test, the ventilation system and the heating system were turned off. The interior and exterior sun protection were not used during the test.

\subsubsection{On-board monitoring}

The following parameters were measured, each $15 \mathrm{~min}$, while the building was occupied:

- Temperature $\left({ }^{\circ} \mathrm{C}\right)$, relative humidity $(\%)$ and $\mathrm{CO}_{2}$ (ppm) in the bathroom, the living room and the guest room (Fig. 7)

- Pulsed air temperature of the mechanical ventilation $\left({ }^{\circ} \mathrm{C}\right)$

- Gas: total, hot water, cooking $(\mathrm{kWh})$

- Electricity: total, ventilation, lighting $(\mathrm{kWh})$

- Water: total and hot water $\left(\mathrm{m}^{3}\right)$

- Solar thermal panel production (kWh)

Note: The last two last measurements were not used in our study. 


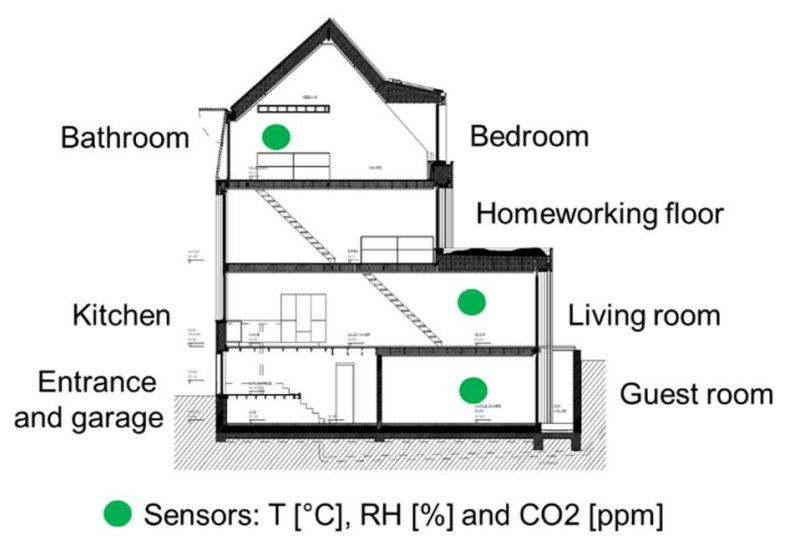

Fig. 7. Sensor positions

External temperature $\left({ }^{\circ} \mathrm{C}\right)$ and Solar radiation $\left(\mathrm{W} / \mathrm{m}^{2}\right)$ were downloaded from a free meteorological database [12].

\section{Results}

\subsection{Statistical analysis}

\subsubsection{Adapted co-heating}

The following figure (Fig. 8) plots the measured data during the adapted co-heating.

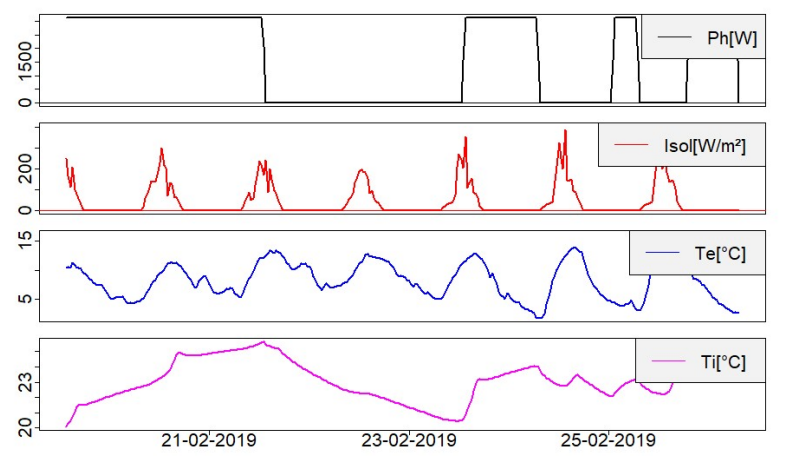

Fig. 8. Time plot of the heating power, the solar radiation, the external temperature and internal temperature.

The average indoor temperature was approx. $23.0^{\circ} \mathrm{C}$, the average outdoor temperature was approx. $8.4^{\circ} \mathrm{C}$, the average solar radiation during the day was approx. $98 \mathrm{~W} / \mathrm{m}^{2}$ and the average heating power was approx. 1619 W.
The following boxplots show more details about the statistical distribution.

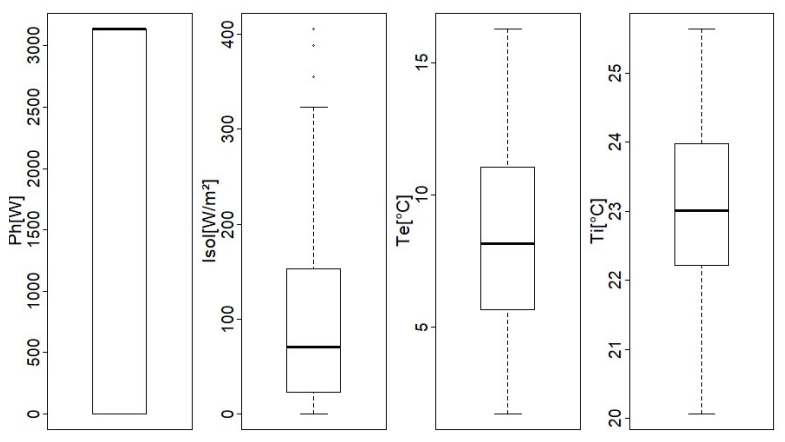

Fig. 9. Statistical distribution of adapted co-heating data

The electrical heating is switched off $50 \%$ of the time, as expected by the signal. The external solar radiation is low comparing the heating power (on average, less than 5 $\%$ ). In addition, an analysis of the sun curve shows that there is very little direct sunlight on windows during the measurements period. We conclude that the solar radiation had little impact on the indoor temperature during measurements.

For all measurements, the average indoor temperature is above $20^{\circ} \mathrm{C}$, however the requirement about the homogeneous indoor temperature was not respected. Moreover, the difference between the indoor and outdoor temperature was in average greater than $10 \mathrm{~K}$, excepted during $10 \%$ of the measurement periods.

\subsubsection{Occupied data analysis}

The following figure (Fig. 10) plots the data monitored during the selected period, from 10/11/2015 to 10/02/2016.
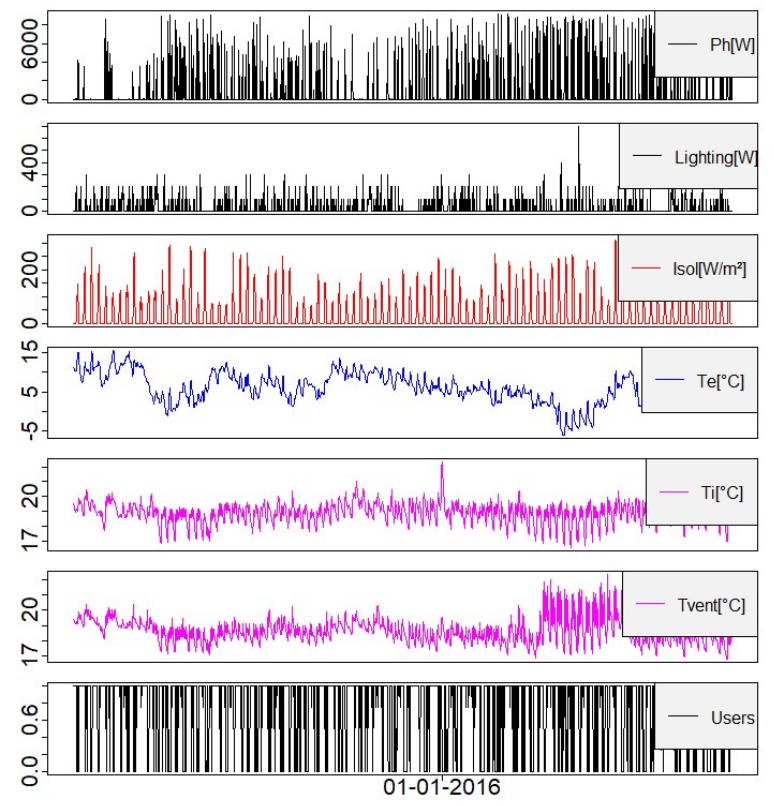

Fig. 10. Time plot of the on-board measurements

The average indoor temperature was approx. $18.9^{\circ} \mathrm{C}$ and the average outdoor temperature was approx. $6.2^{\circ} \mathrm{C}$. 
The indoor temperature might seem low, but it should be noted that the guest room is not heated on a regular basis.

The average solar radiation during the day was approx. $88 \mathrm{~W} / \mathrm{m}^{2}$ and the average heating power was approx. $994 \mathrm{~W}$ when considered over the whole period. When only the periods where the heating was on are considered ( $\pm 20 \%$ of the time), the average heating power was approx. $4481 \mathrm{~W}$.

The following boxplots show more details about the statistical distribution.
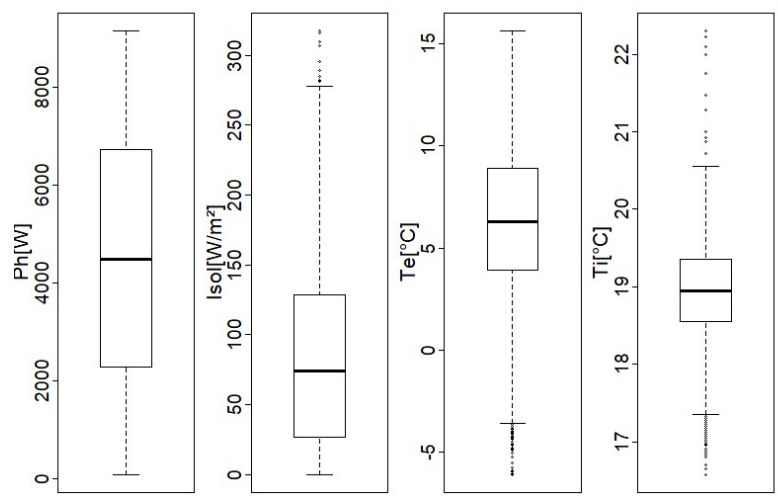

Fig. 11. Statistical distribution of the on-board monitoring.

The occupancy rate resulting from the algorithm described in $\S 3.2 .2$ is approx. $74 \%$. The following plot (Fig. 12) focuses on the results of the detection algorithm in the guest room, over a few days.
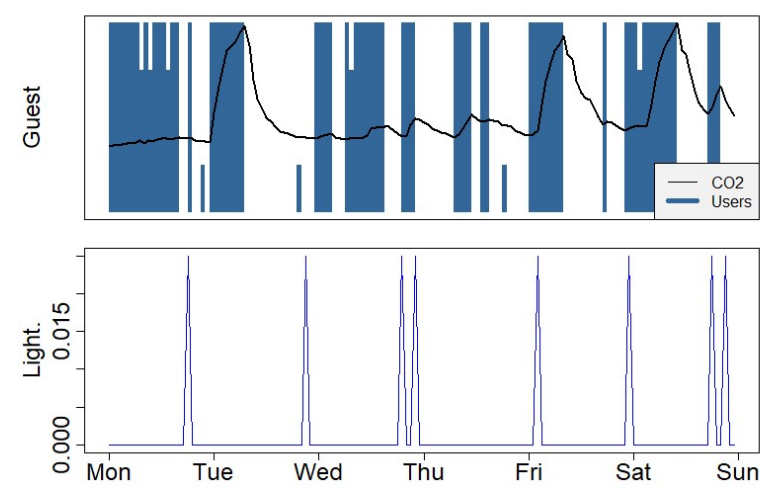

Fig. 12. Occupancy profile in the guest room based on $\mathrm{CO}_{2}$ concentrations in parallel with light activation

Certain occupancy periods are consistent with sleeping hours (during night), as expected in a bedroom. Moreover, at night lighting is switch on/off when occupancy starts and ends, as usual when entering and leaving a room. For the others moment, presences can be explained by some activities of the occupants during the day. Unfortunately, we don't have enough information's to validate further the algorithm we used.

\subsection{Measured HLC}

\subsubsection{Adapted co-heating}

The RC model used to analyze the data collected across the adapted co-heating test is similar to the one given by
Fig. 2. The only difference is that solar radiation is neglected. Indeed, due to the orientation of the main windows (east-west) and the period during which the measurements took place (February), solar radiation can be neglected in the RC model.

CTSM-R was used to identify the parameters $R_{i}, R_{e}, C_{i}$ and $\mathrm{C}_{\mathrm{w}}$. Inputs and output of the $\mathrm{RC}$ model are the measurements of $\mathbf{T}_{\mathbf{i}}, \mathrm{T}_{\mathrm{e}}, \mathrm{I}_{\mathrm{sol}}$ and $\Phi_{\mathrm{Ph}}$ plot in Fig. 8.

Then, a HLC of $106.3 \mathrm{~W} / \mathrm{K}$ was estimated with an uncertainty of $+/-5 \%$ calculated according to the methodology presented in [4].

\subsubsection{Occupied data analysis}

Concerning the data collected during occupancy, the following RC model was used.

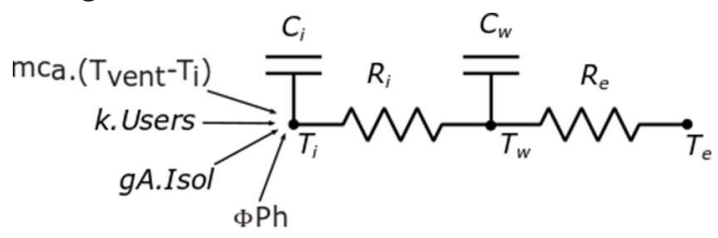

Fig. 13. RC model used to analyze data monitored during occupancy between 11/2015 and 02/2016.

For this on-board monitoring,

$$
\Phi \mathrm{Ph}=\mathrm{Ph}+\text { Lighting }
$$

Where $\mathrm{Ph}$ is the energy used by the heating system multiplied by his efficiency $(85 \%)$ and Lighting is the energy consumed by the lighting systems.

Remember that the inputs and output of the model are the measurements of $\mathbf{T}_{\mathbf{i}}, \mathbf{T}_{\mathbf{e}}, \mathbf{I}_{\mathbf{s o l}}, \boldsymbol{\Phi}_{\mathbf{P h}}, \mathbf{T}_{\mathbf{v e n t}}$ and Users (Fig. $10)$.

CTSM-R was used to identify the parameters $\dot{m} . c_{a}, k$, $\mathrm{gA}, \mathrm{R}_{\mathrm{i}}, \mathrm{R}_{\mathrm{e}}, \mathrm{C}_{\mathrm{i}}$ and $\mathrm{C}_{\mathrm{w}}$. Then, based on 3 months of data, a HLC of $120.8 \mathrm{~W} / \mathrm{K}$ was estimated with an uncertainty of $+/-8 \%$.

The following plot (Fig. 14) shows the reproducibility of the results where:

- the duration of the data used to resolve the RC model is shortened to 1 month.

- each starting date is shifted by 15 days.

HLC with 30 days of data [W/K]

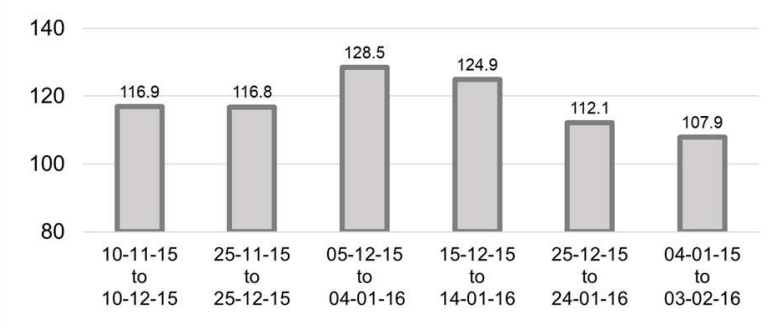

Fig. 14. Reproducibility analysis

Results vary between 107.9 and $128.5 \mathrm{~W} / \mathrm{K}$ and are in average equal to $117.9 \mathrm{~W} / \mathrm{K}$. Compared to the HLC computed from 3 months of data $(120.8 \mathrm{~W} / \mathrm{K})$, results vary between $-11 \%$ to $+6 \%$. 


\subsubsection{Protocols comparison}

All the results presented in this paper are combined into the following figure (Fig. 15).

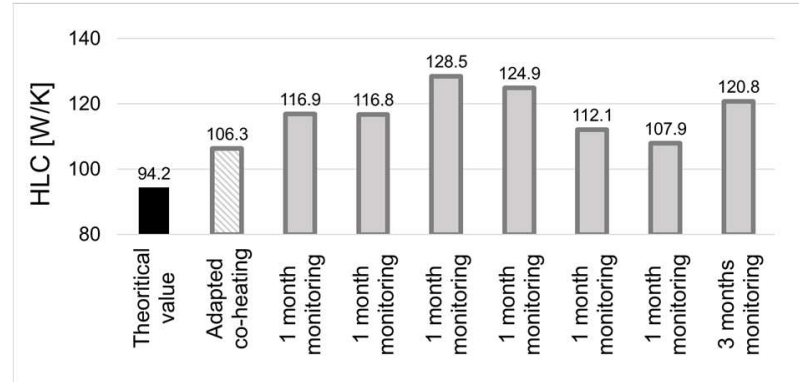

Fig. 15. All HLC estimations

In the theoretical method, the heat exchange between party walls is not considered $(b=0)$. However, heat exchanges are present as soon as the indoor temperature of the adjacent spaces differs. Theoretical value could vary between $94.2 \mathrm{~W} / \mathrm{K}$ and $139.4 \mathrm{~W} / \mathrm{K}$ depending of the reduction factor we apply on that wall (0 to 1 ), (see equation 20). Considering losses to adjoining spaces with an equilibrium temperature equal to $18^{\circ} \mathrm{C}$ or a b factor equal to 0.32 , the theoretical value would be $108.3 \mathrm{~W} / \mathrm{K}$.

on other hand, the proportion of losses due to infiltration and thermal bridges is low ( $\pm 3 \%)$. Airtightness was measured thanks to a blower door test and thermal bridges were calculated in detail. Assumptions related to these losses can't make the difference between the theoretical and the measured values. Thus, the difference between the theoretical value $(94.2 \mathrm{~W} / \mathrm{K})$ and the adapted co-heating value $(106.3 \mathrm{~W} / \mathrm{K})$ should be mainly due to heat exchange with the party wall that would contribute around $13 \%$ of the losses (instead of $0 \%$ ).

Considering that adapted co-heating test gives the reference value, the results obtained with data monitored during occupancy overestimate the expected HLC, from $+2 \%$ to $+21 \%$. The variability of the HLC computed from on-board monitoring can be explained by the assumptions made in the RC model (Fig. 13). Firstly, the occupancy profile determined by the algorithm is a gross simplification of real occupancy. Secondly, infiltration losses, ventilation rate and solar heat gain factor are assumed to be constant. A more detailed monitoring combined with a more complex RC model should improve the reproducibility of the results.

\section{Conclusion}

Two protocols to determine the HLC based on in-situ measurements were compared: adapted co-heating test and on-board monitoring.

Theoretical value seems to under evaluate the real building envelope performance. Indeed, all measured HLC are between $+13 \%$ to $+36 \%$ higher than the theoretical value.

HLC computed from the adapted co-heating is $13 \%$ higher compare to theoretical value. This difference could mainly be explained by the heat exchange across the party wall. Thus, the adapted co-heating result seems to be more representative of the real performance of the building envelope. That value can be used as a reference for in-situ evaluation.

HLC computed from on board monitoring is between $+2 \%$ to $+21 \%$ higher compare to the adapted co-heating result. This difference is mainly explained by the complexity to analyze data monitored during occupancy.

Results presented in this paper are encouraging and on-board monitoring combined with RC model could be a good way to give an indicator of the performance of the building during occupancy.

However, the full approach should be validated on a freestanding house in order to remove the noise due to heat exchange with adjacent houses. Moreover, the onboard monitoring should measure more parameters. As for example, the wind speed and orientation, the ventilation rate and a better indicator of the presence.

This paper is written as part of the prenormative project "CoDyNi 2" funded by the Federal Public Service Economy, SMEs, Self-employed and Energy.

The authors are grateful to Brussels Environment for sharing the monitoring data of the case study presented in this paper.

\section{References}

[1] G. Bauwens, S. Roels, PhD Theses, In Situ Testing of a Building's Overall Heat Loss Coefficient Embedding Quasi-stationary and Dynamic Tests in a Building Physical and Statistical Framework, (2015)

[2] TC89/WG13, In-situ thermal performance of construction products, building elements and structures

[3] J. Wingfield, D. Johnston, D. Miles-Shenton, M. Bell, Leeds Metropolitan University, Whole House Heat Loss Test Method (Coheating), (2010)

[4] R. Jack, D. Loveday, D. Allinson \& K. Lomas, Build. Res. \& Inf., First evidence for the reliability of building co-heating tests, 46:4, 383-401, (2018)

[5] S. Thébault, R. Bouchié, En. \& Build., Refinement of the ISABELE method regarding uncertainty quantification and thermal dynamics modelling, 178, 182-205, (2018)

[6] IEA- EBC Annex 71: Building energy performance assessment based on in situ measurements

[7] D. Farmer, D. Johnston, \& D. Miles-Shenton, En. and Build., Obtaining the heat loss coefficient of a dwelling using its heating system (integrated coheating), 117, 1 - 10, (2016).

[8] G. Masy1, S. Hincque, A. Dawans, Assessment of the Energy Performance of building facades through different testing conditions and analysis techniques, SSB, (2018) 
[9] ISO 8996: Ergonomics of the thermal environment Determination of metabolic rate, ISO/TC 159/SC 5 - Ergonomics of the physical environment

[10] J.A. Diaz, M.J. Jiménez, Appl. Energy, Experimental assessment of room occupancy patterns in an office building. Comparison of different approaches based on $\mathrm{CO}_{2}$ concentrations and computer power consumption, 199, 121-141 (2017)

[11] https://environnement.brussels/thematiques/villedurable/sinspirer-des-batiments-exemplaires

[12] http://www.soda-pro.com/webservices/radiation/cams-radiation-service 